\title{
SATISFAÇÃO FAMILIAR EM UNIDADES DE CUIDADOS INTENSIVOS: REVISÃO INTEGRATIVA DA LITERATURA
}

Family satisfaction in intensive care units: integrative literature review

Satisfacción familiar en unidades de cuidados intensivos: revisión bibliográfica integradora

\author{
Maria João Mar*, Nuno Esteves ${ }^{* *}$, Ana Sabrina Sousa ${ }^{* * *}$
}

\section{RESUMO}

Enquadramento: a dificuldade para avaliar a satisfação do doente numa unidade de cuidados intensivos (UCI) determina que para aferir a qualidade dos cuidados nestas unidades seja considerada a satisfação familiar. Objetivos: verificar a viabilidade do questionário 'FS-ICU-24' enquanto instrumento para avaliar a satisfação familiar; identificar níveis de satisfação através do questionário com enfase nos itens onde os níveis são mais baixos e estratégias para aumentar a satisfação. Metodologia: revisão integrativa com análise de artigos publicados entre 2014 e 2019 na base de dados EBSCO e no FSICU.org. Descritores: Satisfação do doente; Cuidados intensivos; FS-ICU-24. Para sistematizar a informação, foi elaborada uma tabela com os pontos: autores e ano; país, objetivos do estudo; resultados e participantes. Resultados: o FSICU-24 é um bom instrumento para aferir a satisfação familiar na UCI. Os níveis de satisfação familiar são altos (Superiores a 70\%). Nas questões de resposta aberta, familiares indicam aspetos menos positivos como o ambiente físico e a comunicação. Algumas das hipóteses de melhoria sugeridas são proporcionar privacidade na sala de espera e treino à equipa em comunicação. Conclusão: a revisão integrativa evidencia que o FS-ICU-24 permite aferir a satisfação familiar e identificar pontos a melhorar na UCl.

Palavras-Chave: satisfação do doente; cuidados intensivos; FS-ICU-24

*Enfermeira no Serviço de Transplantes de Medula Óssea no Instituto Português de Oncologia do Porto, com mestrado com especialização em Enfermagem MédicoCirúrgica.

${ }^{* *}$ Enfermeiro Especialista em Enfermagem Médico-Cirúrgica no serviço de Medicina Intensiva do Centro Hospitalar de São João.

${ }^{* * *} \mathrm{PhD}$, Centro Hospitalar S. João, Universidade Católica Portuguesa.

Como Referenciar:

Mar, M.J., Esteves, N., \& Sousa, A.S. (2020). Satisfação familiar em cuidados intensivos: revisão integrativa da literatura Revista de Investigação \& Inovação em Saúde, 3(1), 67-77. doi.10.37914/riis.v3i1.61

\section{ABSTRACT}

Background: due to the difficulty of evaluating and intensive care unit (ICU) patient's satisfaction, family satisfaction must be taken into consideration when assessing the quality of the care provided in these units. Objectives: evaluate the feasibility of the quiz 'FS-ICU-24'as an instrument to assess family satisfaction; identify satisfaction levels through a questionnaire with an emphasis on items where satisfaction levels are lower and strategies to increase it. Methodology: an integrative review that analyzes relevant articles published in EBSCO and in FSICU.org data bases between 2014 and 2019. Key words: patient satisfaction; critical care; FS-ICU-24. In order to achieve systematized information, a table with the following points was prepared: authors and publication date, country, study objectives, results and participants. Results: 'FS-ICU-24' is a good instrument to assess family satisfaction in the ICU. Family satisfaction levels are high (above 70\%). In openended questions relatives refer less positive aspects such as the physical environment and communication. Some improvement suggestions are the possibility to increase privacy at waiting rooms and provide communication skills training to the staff. Conclusion: the integrative review shows that the FS-ICU-24 allows family satisfaction assessment and identification of improvements points in ICU.

Keywords: patient satisfaction; critical care; FS-ICU-24

\section{RESUMEN}

Marco contextual: la dificultad para evaluar la satisfacción del paciente internado en una unidad de cuidados intensivos $(\mathrm{UCl})$ determina que para averiguar la calidad de los cuidados prestados en este tipo de unidades sean consideradas las necesidades y satisfacción familiar. Objetivos: comprobar la viabilidad del 'FS-ICU-24' como instrumento para evaluar la satisfacción familiar; identificando los ítems donde esta satisfacción es más baja y qué estrategias se adoptan para aumentar. Metodología: revisión integradora donde se analizaron artículos (en inglés) publicados entre el 2014 y el 2019 en la base de datos EBSCO y sitio web organizacional FSICU.org. Se utilizaron como palabras clave: satisfacción del pacient, cuidados intensivos, FS-ICU-24. Para sistematizar esta información artículos se elaboró un instrumento con informaciones tales como, autores y año de publicación, pais, objetivo de estúdio, resultados y participantes. Resultados: el 'FS-ICU-24' es un bueno instrumento para averiguar la satisfacción familiar en la UCl. Los niveles de satisfacción familiar en general son altos (superiores que $70 \%$ ). En la respuesta abierta del cuestionario, los familiares indican aspectos menos positivos relacionados con el ambiente físico y con la comunicación, y también son sugeridas hipótesis de mejoría cómo proporcionar más privacidad en la sala de espera y capacitar al equipo de comunicación. Conclusión: la revisión integradora muestra que la FS-ICU24 permite medir la satisfacción familiar e identificar puntos para mejorar en la $\mathrm{UCl}$.

Palabras clave: satisfacción del paciente; cuidados intensivos; FS-ICU-24 


\section{INTRODUÇÃO}

Avaliar as necessidades e o grau de satisfação dos familiares de doentes internados na UCI torna-se parte essencial dos cuidados dos profissionais de saúde, que para além da prestação de cuidados, estes têm como função diminuir a dor e o sofrimento dos familiares dos doentes na Unidade de Cuidados Intensivos (UCl) (Neves, Dantas, Bitencourt, Vieira, Magalhães, \& Teles, 2009).

Dado que $20 \%$ dos doentes admitidos na UCI não sobrevivem e os restantes são frequentemente incapazes de recordar e avaliar a sua experiência, mensurar a satisfação do doente internado neste tipo de unidades é um desafio que ainda se coloca na atualidade (Ferrando, et al., 2019).

Esta dificuldade em avaliar a satisfação do doente internado na $\mathrm{UCl}$ conduz inevitavelmente a que para aferir a qualidade dos cuidados prestados na unidade de cuidados intensivos, sejam forçosamente consideradas as necessidades e a satisfação familiar (Tastan, Iyigun, Ayhan, Kılıckaya, Yılmaz, \& Kurt, 2013).

A abordagem à família do doente internado tem sido alvo de inúmeras pesquisas ao longo dos anos, no entanto, na especificidade do doente internado na $\mathrm{UCl}$, esta questão torna-se particularmente complexa, uma vez que todo o equilíbrio familiar é alterado pela doença grave e pela assunção de um evento catastrófico gerador de sentimentos medo, choque e descrença. Toda a família, assim como o doente sofrem com a rutura da sua rotina e do seu ambiente, pela perceção da degradação familiar e da descontinuidade da sua história de vida, onde o tratamento impessoal prestado pela equipa, a exclusão da rotina hospitalar e a uniformização no tratamento dos doentes, podem gerar na família a sensação de perda de controle, despersonalização, exacerbação dos mecanismos de defesa, sentimento de abandono e confusão cognitiva (Ismael, 2010). Neves, Schwartz, Guanilo, Amestoy, Mendieta \& Lise (2018) indicam que a avaliação da satisfação dos familiares na fase da admissão do doente na $\mathrm{UCI}$ permitiria à equipa a adequação da sua prestação de cuidados, tornando-a mais direcionada e visando melhores resultados, tanto para o doente como para os familiares que o acompanham.

Neste sentido, desde 1970 que têm surgido vários questionários para identificar as necessidades da família e a satisfação com os cuidados prestados nas unidades de cuidados intensivos, sendo o 'Family Satisfaction in the intensive care unit - 24 (FS-ICU-24)' o instrumento mais amplamente usado (Kim, et al., 2017).

O 'Family Satisfaction with Care in the Intensive Care Unit (FS-ICU)' foi desenvolvido no Canadá e inclui os conceitos satisfação com o cuidado do doente, qualidade no fim de vida, necessidades dos familiares e satisfação/ insatisfação com decisões médicas (Heyland, \& Tranmer, 2001). A versão inicial era composta por 34 questões, mas anos mais tarde, o questionário passou por uma revisão, a qual originou a versão atual com 24 questões. Esta adaptação recebeu colaboração de pesquisadores dos Estados Unidos, sendo validada para ambos os países Canadá e Estados Unidos da América. Em Portugal o instrumento encontra-se traduzido e sua utilização está autorizada pelos autores. A partir desta revisão, definiu-se um score para mensurar a satisfação em relação aos cuidados, um score para a participação no processo de tomada de decisão e um score de 
Satisfação familiar em unidades de cuidados intensivos: revisão integrativa da literatura

satisfação geral que engloba o cuidado e a tomada de decisão. Além dos três itens avaliados, o instrumento também prevê um score e questões para avaliar familiares de pacientes de não sobreviventes em relação aos cuidados no final de vida e óbito na UCI. As questões são respondidas com base numa escala de Likert, quantificada de 1 a 5 em que 1- "Excelente", 2- "Muito Bom", 3- "Bom", 4- "Razoável" e 5- "Mau". Existe ainda uma sexta opção "N/A- Não se aplica", para situações em que o inquirido considere que a questão não se enquadra no seu caso (Wall, Engelberg, Downey, Heyland, \& Curtis, 2007).

A revisão integrativa foi realizada para dar resposta aos seguintes objetivos: verificar a viabilidade do questionário 'FS-ICU-24' enquanto instrumento para avaliar a satisfação familiar; identificar os itens onde os níveis de satisfação familiar são mais baixo e quais as estratégias adotadas para aumentar o nível de satisfação familiar.

\section{PROCEDIMENTOS METODOLÓGICOS DE REVISÃO}

A revisão integrativa é uma metodologia que proporciona a síntese de conhecimento e a incorporação da aplicabilidade de resultados de estudos significativos na prática. Diante da necessidade de uma prática baseada na evidência, a revisão integrativa tem sido apontada como uma ferramenta ímpar no campo da saúde, pois sintetiza as pesquisas disponíveis sobre determinada temática e direciona a prática fundamentando-se em conhecimento científico (Souza, Silva \& Carvalho, 2010).

A revisão integrativa no âmbito da satisfação familiar na UCI foi realizada em dezembro de 2019 nas bases de dados EBSCO e FSICU.org com definição das palavras-chave Patient Satisfaction, Critical Care, FSICU-24 e com utilização do recurso operador booleano AND. O termo 'FS - ICU - 24' apesar de não ser um termo MESH (Medical Subject Headings) faz sentido ser incluído nesta revisão, uma vez que é objetivo desta pesquisa verificar a satisfação familiar de doentes internados na $\mathrm{UCl}$ com o instrumento questionário 'FS-ICU-24'. Como critérios de inclusão foram definidos ano 2014-2019 e texto integral.

O processo de pesquisa e seleção de artigos a incluir na revisão integrativa encontra-se ilustrado no diagrama PRISMA (Figura 1). O mesmo decorreu da seguinte forma, foram introduzidas as palavras-chave na base de dados EBSCO e no site da organização FSICU.org. Inicialmente foram obtidos treze artigos na base de dados EBSCO e um artigo no FSICU.org. No processo de seleção foi definido como critério de inclusão, texto integral, assim foram excluídos três artigos. Na fase da inclusão, por inadequação ao tema foram excluídos cinco artigos. $O$ artigo obtido da FSICU.org manteve-se ao longo do processo de seleção e inclusão. 


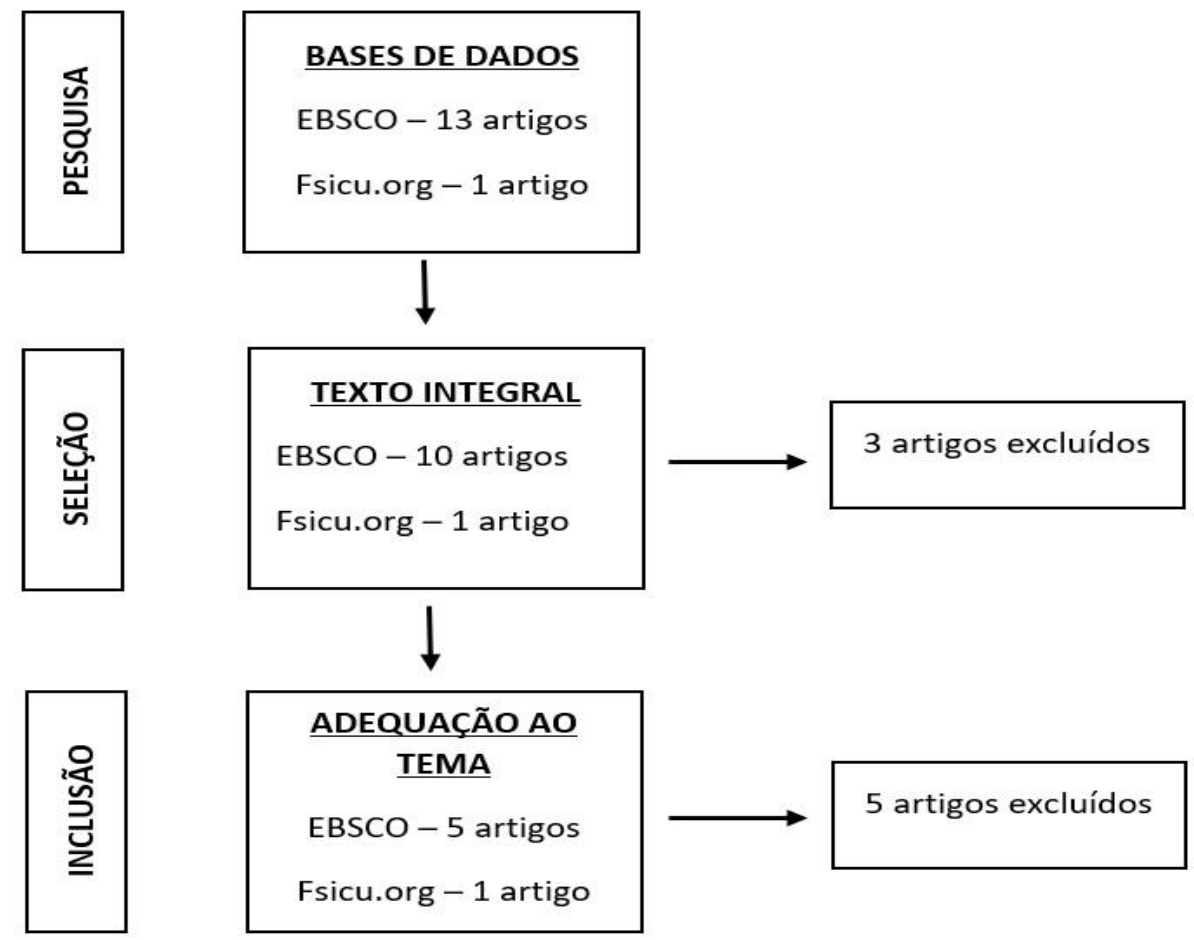

Figura 1

Diagrama PRISMA

\section{RESULTADOS}

Os artigos incluídos nesta revisão integrativa revelam pontos comuns, nomeadamente no que diz respeito à robustez do questionário, aos pontos em que a satisfação familiar apresenta scores mais baixos e também nas propostas para aumento da satisfação familiar. No Quadro 1 apresentam-se os artigos selecionados para a revisão integrativa, destacando-se os seguintes aspetos: autores e ano; país, objetivos do estudo; resultados e participantes.

\section{Quadro 1}

Síntese de artigos incluídos na revisão integrativa

\begin{tabular}{|c|c|c|c|c|}
\hline Autores e Ano & País & Objetivo do estudo & Resultados & Participantes \\
\hline $\begin{array}{l}\text { Ferrando et } \\
\text { al. } \\
2019\end{array}$ & Reino Unido & $\begin{array}{l}\text { Aferir a satisfação } \\
\text { familiar com as } \\
\text { unidades de cuidados } \\
\text { intensivos em UK } \\
\text { utilizando } \\
\text { questionário FS-ICU- } \\
24\end{array}$ & $\begin{array}{l}\text { O FS-ICU-24 pode ser usado para aferir a } \\
\text { satisfação familiar, mas deve ser ajustado às } \\
\text { características das diferentes famílias; } \\
\text { O nível de satisfação é alto, } \\
\text { contudo o facto de haver uma cauda longa } \\
\text { significa que há resultados muito baixos; } \\
\text { Familiares de não sobreviventes apresentam } \\
\text { níveis de satisfação mais altos do que } \\
\text { familiares de sobreviventes; } \\
\text { Para familiares de não sobreviventes só as } \\
\text { caraterísticas do doente eram significativas, }\end{array}$ & $\begin{array}{l}7173 \text { membros da família de } 4615 \\
\text { doentes internados na UCI por mais } \\
\text { de } 24 \text { horas entre maio de } 2013 \text { e } \\
\text { junho de } 2014\end{array}$ \\
\hline
\end{tabular}




\begin{tabular}{|c|c|c|c|c|}
\hline & & & $\begin{array}{l}\text { para familiares de sobreviventes tanto as } \\
\text { características do doente como da família } \\
\text { eram determinantes na satisfação familiar. } \\
\text { Este estudo apresenta como limitação } \\
\text { enviesamentos, o facto de só incluir } \\
\text { familiares de doentes internados há mais de } \\
24 \text { horas, pode excluir os familiares de } \\
\text { doentes graves que morrem nas primeiras } 24 \\
\text { horas e ainda diferenças na evolução dos } \\
\text { doentes cujos familiares responderam ao } \\
\text { questionário, possível bies. }\end{array}$ & \\
\hline $\begin{array}{l}\text { Tastan et al. } \\
2013\end{array}$ & Turquia & $\begin{array}{l}\text { Testar a versão Turca } \\
\text { do FS-ICU-24 }\end{array}$ & $\begin{array}{l}\text { O FS-ICU-24 pode ser usado para aferir a } \\
\text { satisfação familiar; } \\
\text { Os maiores níveis de satisfação familiar estão } \\
\text { relacionados com os cuidados prestados } \\
\text { pelos enfermeiros e pelos médicos ao } \\
\text { doente; } \\
\text { Os níveis mais baixos de satisfação estão } \\
\text { relacionados com: } \\
\text { - Informações dadas pelos enfermeiros } \\
\text { acerca do estado do doente; } \\
\text {-Condições da sala da sala de espera da } \\
\text { unidade de cuidados intensivos. } \\
\text { Como limitação este estudo apresenta o } \\
\text { facto de as expectativas dos familiares } \\
\text { influenciarem a satisfação com os cuidados. }\end{array}$ & $\begin{array}{l}120 \text { familiares de doentes } \\
\text { internados em departamentos de } \\
\text { anestesia e reanimação, por mais de } \\
48 \text { horas, entre maio e dezembro de } \\
2008\end{array}$ \\
\hline $\begin{array}{l}\text { Clark et. al } \\
2016\end{array}$ & $\begin{array}{l}\text { Estados } \\
\text { Unidos da } \\
\text { América }\end{array}$ & $\begin{array}{l}\text { Implementar um } \\
\text { instrumento para } \\
\text { avaliação a satisfação } \\
\text { familiar na UCl; } \\
\text { Mensurar a satisfação } \\
\text { familiar e fazer } \\
\text { recomendações com } \\
\text { base nos resultados } \\
\text { obtidos. }\end{array}$ & $\begin{array}{l}\text { O FS-ICU-24 tem viabilidade para mensurar a } \\
\text { satisfação familiar na UCl; } \\
\text { Media de satisfação geral } 72.24 \% \text {; } \\
\text { Media de satisfação com os cuidados } \\
\text { prestados } 70.87 \% \text {; Media de satisfação com a } \\
\text { tomada de decisão } 72.03 \% \text {; } \\
\text { Pontos comuns descritos pelas famílias em } \\
\text { texto livre: } \\
\text {-Comentários positivos acerca da equipa, dos } \\
\text { cuidados e do conforto que a equipa } \\
\text { providencia; } \\
\text { Comentários negativos: necessidade de } \\
\text { melhor comunicação com a equipa; ambiente } \\
\text { físico - sugestões de melhoria da sala de } \\
\text { espera e estacionamento. } \\
\text { Como limitação este estudo apresenta o } \\
\text { facto de os questionários serem recolhidos } \\
\text { enquanto o doente está internado o que } \\
\text { pode ocasionar falsos positivos e ainda o a amostra não ser representativa da }\end{array}$ & $\begin{array}{l}39 \text { membros da família de doentes } \\
\text { internados na UCl entre outubro de } \\
2014 \text { e dezembro de } 2014\end{array}$ \\
\hline
\end{tabular}


Satisfação familiar em unidades de cuidados intensivos: revisão integrativa da literatura

\begin{tabular}{|c|c|c|c|c|}
\hline & & & $\begin{array}{l}\text { população e ainda o facto de a amostra ser } \\
\text { pequena. }\end{array}$ & \\
\hline $\begin{array}{l}\text { Lyes et al. } \\
2019\end{array}$ & Reino Unido & $\begin{array}{l}\text { Avaliar a eficácia das } \\
\text { estratégias adotadas } \\
\text { para aumentar a } \\
\text { satisfação familiar } \\
\text { tendo por base os } \\
\text { resultados do FS-ICU- } \\
24\end{array}$ & $\begin{array}{l}\text { O FS-ICU-24 é um instrumento válido para } \\
\text { avaliar a satisfação familiar } \\
\text { A maior parte (63\%) dos respondentes } \\
\text { apresentam respostas que correspondem a } \\
\text { satisfação } 80 \% \text { ou superior; } \\
\text { As respostas abertas foram codificadas em } \\
\text { seis tópicos: Ambiente físico; cuidado ao } \\
\text { doente e à pessoa; cuidado aos membros da } \\
\text { família; comunicação e tomada de decisão; } \\
\text { cuidados à equipa; internamento hospitalar. } \\
\text { Para os tópicos criados foram propostas } \\
\text { hipótese de melhoria. } \\
\text { Uma limitação do estudo prende-se com o } \\
\text { facto de os tópicos poderem não abranger } \\
\text { exaustivamente todas as atividades de } \\
\text { melhoria da qualidade. }\end{array}$ & $\begin{array}{l}1855 \text { membros da família de } \\
\text { doentes internados na UCl por mais } \\
\text { de } 48 \text { horas }\end{array}$ \\
\hline $\begin{array}{l}\text { Kim et al. } \\
2017\end{array}$ & Coreia do Sul & $\begin{array}{l}\text { Traduzir para coreano } \\
\text { e validar versão } \\
\text { coreana do FS-ICU- } 24\end{array}$ & $\begin{array}{l}\text { A versão coreana do FS-ICU } 24 \text { constitui um } \\
\text { bom instrumento para avaliar a satisfação } \\
\text { familiar. } \\
\text { A média da satisfação geral foi de } 75.44 \% \text {. Os } \\
\text { participantes estavam mais satisfeitos com } \\
\text { serem consideradas as suas necessidades e } \\
\text { menos satisfeitos com o ambiente físico da } \\
\text { UCl e da sala de espera. } \\
\text { Como limitação apresenta o facto de as } \\
\text { respostas serem maioritariamente de } \\
\text { familiares de sobreviventes. }\end{array}$ & $\begin{array}{l}200 \text { membros da família de } 176 \\
\text { doentes internados na UCI por mais } \\
\text { de } 48 \text { horas Family; intensive care } \\
\text { units; satisfaction; validation } \\
\text { studies }\end{array}$ \\
\hline $\begin{array}{l}\text { Bjørg Dale; } \\
\text { Gro Frivold } \\
2018\end{array}$ & Noruega & $\begin{array}{l}\text { Testar a tradução e } \\
\text { explorar as } \\
\text { propriedades } \\
\text { psicométricas de } \\
\text { versão norueguesa do } \\
\text { questionário }\end{array}$ & $\begin{array}{l}\text { O FS-ICU-24 apresenta propriedades } \\
\text { psicométricas o que indica que é um } \\
\text { instrumento adaptado para aferir a } \\
\text { satisfação familiar } \\
\text { Uma limitação deste estudo é o facto de a } \\
\text { amostra ser pequena, o impacto cultural e o } \\
\text { facto de os questionários terem sido colhidos } \\
\text { numa única data. }\end{array}$ & $\begin{array}{l}123 \text { membros da família de doentes } \\
\text { internados na } \mathrm{UCl} \text { por mais de } 24 \\
\text { horas }\end{array}$ \\
\hline
\end{tabular}

Ferrando et al. (2019) verificaram que da aplicação do 'FS-ICU 24', em geral, o nível de satisfação é alto, contudo o tratamento dos dados evidencia que há uma cauda longa, o que significa que há resultados muito baixos. É ainda percetível que os familiares de não sobreviventes apresentam níveis de satisfação mais altos do que familiares de sobreviventes. Outro ponto verificado pelos autores, é que para familiares de não sobreviventes só as caraterísticas do doente eram significativas, enquanto que para familiares de sobreviventes tanto as características do doente como da família eram determinantes na satisfação familiar. Tastan et al. (2013) decorrente da aplicação do questionário constataram que os maiores níveis de 
satisfação familiar estão relacionados com os cuidados prestados pelos enfermeiros e pelos médicos ao seu familiar. Por outro lado, os níveis mais baixos de satisfação familiar estão associados às informações dadas pelos enfermeiros acerca do estado do doente $\mathrm{e}$ as condições da sala da sala de espera da $\mathrm{UCl}$.

Clark, Milner, Beck, \& Mason, (2016) apontam como média de satisfação geral $72,24 \%$, como média de satisfação com os cuidados prestados de $70,87 \%$ e como média de satisfação com a tomada de decisão de 72,03\%. Da análise das questões de resposta aberta dadas pelas famílias, Clark et al. (2016) identificam comentários positivos acerca da equipa, dos cuidados e do conforto que a equipa providencia e comentários negativos que se relacionam com a necessidade de melhor comunicação com a equipa e sugestões que se relacionam com o ambiente físico, especificamente, sugestões de melhoria da sala de espera e estacionamento.

Neste sentido, Neves et. al (2018) verificaram a necessidade de ampliar as possibilidades de comunicação estabelecidas com os familiares, visto que se trata de um recurso que os aproxima dos profissionais de saúde e facilita a compreensão da condição clínica dos doentes internados, gerando alívio do sofrimento e conforto.

Lyes, Richards-Belle, Connolly, Rowan, Hinton, \& Locock, (2019) verificaram da análise de respostas ao 'FS-ICU 24' que a maior parte das famílias (63\%) apresentam respostas que correspondem a uma satisfação de $80 \%$ ou superior. Relativamente às questões de resposta aberta, estas foram codificadas em seis tópicos: ambiente físico; cuidado ao doente e à pessoa; cuidado aos membros da família; comunicação e tomada de decisão; cuidados da equipa; internamento hospitalar.

Kim et al. (2017) constataram da análise das respostas ao questionário que a média da satisfação geral foi de $75,44 \%$. Contudo, os participantes estavam mais satisfeitos com serem consideradas as suas necessidades e menos satisfeitos com o ambiente físico da sala de espera.

Bjørg, \& Frivold, (2018) aferiram que 'FS-ICU-24' apresenta propriedades psicométricas, o que indica que é um instrumento adaptado para aferir a satisfação familiar.

\section{DISCUSSÃO}

Os artigos incluídos nesta revisão indicam que o 'FSICU-24' apresenta propriedades psicométricas que the conferem viabilidade para mensurar a satisfação familiar em unidades de cuidados intensivos.

Os resultados obtidos indicam que os níveis de satisfação familiar geral são elevados (superiores a $70 \%)$, contudo os familiares de doentes que faleceram apresentam maiores níveis de satisfação do que os familiares de sobreviventes. Estas diferenças parecem estar associadas ao facto de os familiares dos não sobreviventes das unidades de cuidados intensivos terem uma maior envolvência na tomada de decisão no fim de vida. Por outro lado, os familiares de sobreviventes têm de lidar com problemas decorrentes do internamento numa UCl (Ferrando et al., 2019). Especificando a satisfação familiar em termos dos itens descritos no 'FS-ICU-24', os familiares indicam que o que mais os satisfazia eram os cuidados prestados pelos profissionais (enfermeiros e médicos). Por outro lado, os pontos menos satisfatórios eram a frequência da informação que era dada pelos enfermeiros acerca 
do estado do paciente e as condições das salas de espera (Tastan et al., 2013).

Ferrando et al. (2019) apontam ainda que os valores mais baixos de satisfação estão associados à documentação escrita de admissão e alta da unidade de cuidados intensivos e ao rácio enfermeiro/doente.

Lyes et al. (2019) codificaram as questões de resposta aberta presentes no 'FS-ICU-24' em seis tópicos: ambiente físico, cuidados ao paciente e à pessoa, cuidados aos membros da família, comunicação e tomada de decisão, cuidado com a equipa e internamento hospitalar. Os desenvolvimentos destes tópicos remetem para melhorias que conduzem ao aumento dos níveis de satisfação familiar. No tópico ambiente físico os familiares apontam melhoraria da orientação e redução do tédio, a importância de criar um ambiente que permita descansar e dormir e a qualidade da sala de espera. O tópico cuidado ao doente e pessoa remete-nos para os temas conhecer a pessoa, preservar a dignidade e lidar com a alucinação e ventilação assistida. No que diz respeito ao tópico dos cuidados aos membros da família são apontadas a melhoraria do contacto com os familiares doentes, orientação do doente, a prestação de suporte emocional pela equipa e a privacidade no luto. $\mathrm{Na}$ comunicação e tomada de decisão, os temas apontados foram a melhoria do contacto familiar com os médicos, melhoria da comunicação dia-a-dia e melhoria da comunicação com o doente. $O$ tópico cuidado à equipa aponta o treino à equipa em comunicação e o suporte à equipa como temas a desenvolver. Em relação ao internamento hospitalar os temas a desenvolver são a alta dos cuidados intensivos e a comunicação com outros serviços.
Os vários artigos na revisão integrativa apontam para a codificação das questões de resposta aberta e com base nestas respostas, os autores propõem recomendações para aumentar a satisfação familiar, logo, a qualidade dos cuidados no serviço de cuidados intensivos.

Desta forma, as recomendações surgem agrupadas conforme a codificação atribuída pelos autores às questões de resposta aberta.

\section{Ambiente Físico}

Lyes et al. (2019) apontam como recomendações de melhoria na categoria ambiente físico, de forma a ir de encontro ao aumento da satisfação familiar, a colocação de relógios, a utilização de caixotes silenciosos e a troca do toque do telefone.

Por seu lado Clark et al. (2016) propõe nesta categoria, que denominam de medidas de conforto, redesenhar a sala de espera familiar para providenciar confidencialidade e privacidade, identificar sinais e descobrir formas para reduzir o stresse. Propõem ainda, a incorporação dos resultados da evidência científica acerca da disposição do mobiliário, distrações positivas e redução do barulho na saúde da família que visita os cuidados intensivos.

\section{Cuidado ao doente e pessoa}

No sentido de preservar a dignidade do doente, Lyes et al. (2019) sugerem como hipóteses de melhoria usar um sinal que indique que estão a ser prestados cuidados ao doente e o desenvolvimento de formações para a equipa e paciente acerca do impacto da experiência da alucinação.

\section{Cuidado aos membros da família}

Lyes et al. (2019) sugerem como hipóteses de melhoria neste item a promoção do envolvimento da família na 
Satisfação familiar em unidades de cuidados intensivos: revisão integrativa da literatura

prestação de cuidados ao doente, o atendimento dos alarmes rapidamente durante a hora de visita, a equipa médica usar crachás de identificação, a informação do doente ser registada de forma a ser partilhada com o contacto principal e as orientações e regras registadas de forma a serem partilhadas com o contacto principal. Clark et al. (2016) propõem que durante as primeiras 24 após admissão na $\mathrm{UCl}$, a equipa multidisciplinar deverá ser apresentada aos membros da família e mostrado um vídeo que descreva a rotina, os recursos e expetativas. Às 72 horas, um responsável da equipa deve verificar com a família as suas necessidades. Às 96 horas os familiares devem receber um diário de cuidados para ajuda-los a tirar notas, reunir questões, preocupações ou informações que gostavam de partilhar com a equipa. Os autores sugerem ainda que as informações fornecidas pelos familiares sejam levadas em conta e que a família seja encorajada a colaborar nos cuidados, desde que se sintam confortáveis para tal.

\section{Comunicação e tomada de decisão}

No âmbito da comunicação e tomada de decisão as estratégias propostas foram: redesenhar as notas de alta com os familiares, doentes e profissionais de todas as equipas envolvidas nos cuidados ao doente, definir momentos para as reuniões familiares, veicular formação aos enfermeiros no âmbito da comunicação e identificar uma aplicação para computador comunicar com doentes ventilados (Lyes et al. 2019).

Tanstan et al. (2014) enumeram os pontos referidos pelas famílias em resposta aberta e indicam propostas para aumentar a satisfação:

- A satisfação familiar aumenta quando a família é informada acerca do doente de forma completa,

correta e compreensível, ao mesmo tempo que são prestados bons cuidados atempadamente ao doente; Obter informações acerca do seu familiar a qualquer momento do dia mesmo sem perguntar, faz com que os familiares se sintam seguros e bem consigo próprios e faz com que a família se sinta envolvida nos cuidados. As sugestões propostas pelos autores integrados nesta revisão integrativa são corroboradas por Fumis, Nishimoto \& Deheinzelin (2008) que verificaram que a satisfação familiar foi positivamente associada com uma maior frequência de comunicação com a equipa, especialmente com os médicos, clareza sobre o prognóstico e bom relacionamento interpessoal.

\section{Equipa unidade cuidados intensivos}

Lyes et al. (2019) sugerem a criação de um grupo de trabalho na área do delirium, sugerem que os enfermeiros mais experientes sejam incentivados a apoiar os enfermeiros menos experientes e ainda, que os enfermeiros sejam informados acerca dos mecanismos de suporte.

Clark et al. (2016) apontam várias recomendações para a equipa de cuidados intensivos:

$\mathrm{Na}$ admissão na $\mathrm{UCl}$, um elemento designado da equipa reúne com um membro da família a fim de informar acerca dos mecanismos de suporte necessários;

Os profissionais da $\mathrm{UCl}$ receberem formação em comunicação, gestão de conflitos, reconhecimento de sinais de aumento de angústia nos membros da família e cuidados culturalmente adequados;

Médicos e enfermeiros discutirem com cada doente as possibilidades de forma tão consistente quanto possivel; 
Criação de mecanismo para que toda a equipa possa mencionar as suas preocupações com o plano tratamento, descompressão ou luto.

Suporte à família assegurado por equipa multiprofissional.

\section{CONCLUSÃO}

A revisão integrativa realizada evidencia a adequação e aplicabilidade do 'FS-ICU-24' para avaliar a satisfação familiar de doentes internados na UCl.

Os níveis de satisfação familiar obtidos da aplicação do questionário demonstraram valores elevados, havendo, contudo, itens nos quais os níveis de satisfação são mais baixos. Estes itens dizem respeito, sobretudo, à comunicação e ao ambiente físico da unidade e da sala de espera.

As estratégias para o aumento da satisfação familiar apontam para melhorias na comunicação entre a equipa e os familiares dos doentes internados na UCl e ainda a adoção de estratégias que reduzam o ruído, bem como tornar a sala de espera mais acolhedora e que confira maior privacidade aos familiares.

A realização desta revisão integrativa acrescenta valor à prestação de cuidados na medida que sugere estratégias que podem ser aplicadas nos contextos de prática para ir de encontro à satisfação familiar.

Como limitação deste estudo é apontado o facto de não ter sido incluída na revisão integrativa nenhum artigo que espelhasse a realidade portuguesa.

Para terminar, fica a proposta de validação e a adaptação do instrumento para a realidade portuguesa, uma vez que o mesmo apenas se encontra traduzido. E ainda, a aplicação deste questionário amplamente nas unidades de cuidados intensivos e, do tratamento de dados, verificar a aplicabilidade das propostas sugeridas por estes autores para aumentar a satisfação familiar, logo, a qualidade dos cuidados prestados nas unidades de cuidados intensivos em Portugal.

\section{REFERÊNCIAS BIBLIOGRÁFICAS}

Clark, K., Milner, K. A., Beck, M., \& Mason, V. (2016). Measuring Family Satisfaction With Care Delivered in the Intensive Care Unit. Critical Care Nurse, 36(6), e8-e14. doi:10.1111/ijn.12153

Dale, B., \& Frivold, G. (2019). Psychometric Testing of the Norwegian Version of the Questionnaire Family Satisfaction in the Intensive Care Unit (FS-ICU-24). Journal of Multidisciplinary Healthcare, 11, 653-659.

Ferrando, P., Gould, D., Walmsley, E., Richards-Belle, A., Canter, R., Saunders,... Rowan, K. (2019). Family Satisfaction with critical care in the UK: a multicenter cohort study. BMJ Open, 9:e028956. Doi10.1136/bmjopen-2019-028956

Fumis, R., Nishimoto, I., \& Deheinzelin, D. (2008). Families interactions with physicians in the intensive care unit: the impact on family's satisfaction. J Crit Care, 23(3), 281-286

Heyland, D.K., \& Tranmer, J.E. (2001). Measuring family satisfaction with care in the intensive care unit: the development of a questionnaire and preliminary results. J Crit Care, 16(4), 142-149

Ismael, S.A. (2010). família do paciente em UTI. In: Mello, F.J. (org.) Doença e família (pp. 251-258). São Paulo: Casa do Psicólogo

Kim, Y., Min, J., Lim, G., Lee, J., Lee, H., Lee ... Lee, Y. (2017). Transcultural Adaptation and Validation of the Family Satisfaction in the Intensive Care Unit Questionnaire in a Korean Sample. The Korean Journal of Critical Care Medicine. 32(1) 60-69 Retirado de $10.4266 / \mathrm{kjccm} .2016 .00962$.

Lyes, S., Richards-Belle, A., Connolly, B., M. Rowan, K., Hinton, L., \& Locock, L. (2019). Can the UK 24-item family satisfaction in the intensive care unit questionnaire be used to evaluate quality improvement strategies aimed at improving family satisfaction with the ICU? A qualitative study. Journal of the Intensive Care, $0(0)$, 18. https://doi.org/10.1177/1751143719883563

Neves, J., Schwartz, E., Guanilo, M., Amestoy, S., Mendieta, M., \& Lise, F. (2018). Avaliação da Satisfação 
de Familiares de Pacientes Atendidos em Unidades de Terapia Intensiva: Revisão Integrativa. Texto Contexto Enfermagem.27(2),1-12

http://dx.doi.org/10.1590/0104-

070720180001800016

Neves F., Dantas, M.P, Bitencourt, A.G.V, Vieira, O.S., Magalhães, L.T., \& Teles, J.M.M. (2009). Analysis of family satisfaction in intensive care unit. Rev Bras Ter Intensiva. 21(1), 32-37 Available from: http://www.scielo.br/pdf/rbti/v21n1/en_v21n1a05.p df
Souza, M., Silva, M., \& Carvalho, R. (2010). Revisão integrativa: o que é e como fazer. Einstein. 8(1),102-6

Tastan, S., Iyigun, E., Ayhan, H., Kilıckaya, O., Yılmaz, A. A., \& Kurt, E. (2013). Validity and reliability of Turkish version of family satisfaction in the intensive care unit. International Journal of Nursing Practice, 20(3), 320326. doi:10.1111/ijn.12153

Wall, R.J., Engelberg, R., Downey, L., Heyland, D.K., \& Curtis, J.R. (2007). Refinement, scoring and validation of the family satisfaction in the intensive care unit (FSICU) survey. Crit Care Med, 35(1), 271-279 